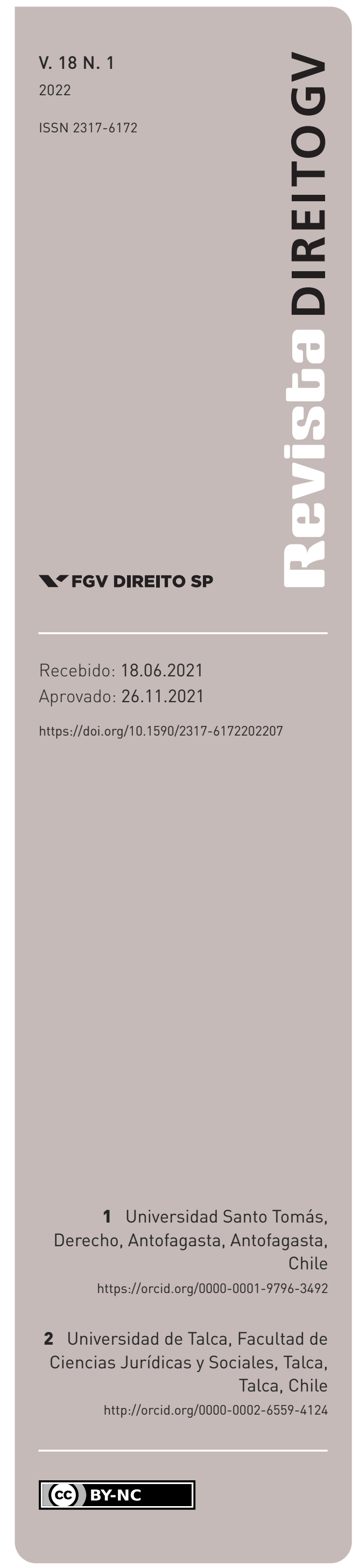

\section{El interés superior en la trayectoria educativa de los niños, niñas y adolescentes con trastorno del espectro autista en Chile}

THE BEST INTEREST OF CHILDREN AND ADOLESCENTS WITH AUTISM SPECTRUM DISORDER IN THE EDUCATIONAL TRAJECTORY IN CHILE

O MELHOR INTERESSE DAS CRIANÇAS E DOS ADOLESCENTES COM TRANSTORNO DO ESPECTRO AUTISTA NA TRAJETÓRIA EDUCACIONAL NO CHILE

Constanza Astudillo Meza y Alexis Mondaca Miranda ${ }^{2}$

\section{Resumen}

El presente trabajo tiene por objeto estudiar las necesidades educativas especiales de los niños, niñas o adolescentes que presentan un trastorno del espectro autista, desde el punto de vista del principio del interés superior aplicado a casos determinados en el contexto de la educación chilena. En este orden de ideas, nos referimos a la admisión de tales niños, niñas o adolescentes en los recintos educacionales considerando el mencionado principio y su aplicación para cada caso en concreto. Como resultado de esta investigación, se determina que debe rechazarse la mera existencia de un trastorno del espectro autista como fundamento de una decisión de no renovación de matrícula y que la derivación a recintos educacionales especiales debe ser una medida excepcional, y se propone una primacía de la "inclusión " por sobre la "integración".

\section{Palabras-clave}

Necesidades educacionales; discriminación educacional; infancia; educación inclusiva; derecho a la educación.

\begin{abstract}
The purpose of this paper is to study the special educational needs of children or adolescents with autism spectrum disorder, from the point of view of the best interest principle. The method used involves the analysis of the corresponding regulations, doctrine and jurisprudence, taking into account the requirements arising from this principle, so as to be able to apply it to specific cases in the context of Chilean education. In this order of ideas, we refer to the admission of such children or adolescents in educational institutions considering the mentioned principle and its application to each specific case. As a result of this research, it is determined that the mere existence of an autism spectrum disorder should be rejected as a basis for a decision of non-renewal of enrollment and that referral to special educational facilities should be an exceptional measure, and a primacy of "inclusion" over "integration" is proposed.
\end{abstract}

\section{Keywords}

Educational needs; educational discrimination; childhood; inclusive education; right to education.

\section{Resumo}

O objetivo deste artigo é estudar as necessidades educacionais especiais de crianças ou adolescentes com transtorno do espectro autista do ponto de vista do princípio do melhor interesse. 0 método utilizado envolve a análise dos regulamentos, da doutrina e da jurisprudência correspondentes, levando em conta as exigências 
decorrentes desse princípio, aplicado a casos específicos no contexto da educação chilena. Nesse contexto, referimo-nos à admissão de tais crianças ou adolescentes em instituições educacionais, considerando o princípio mencionado e sua aplicação em cada caso específico. Como resultado desta pesquisa, é determinado que a mera existência de um transtorno do espectro autista deve ser rejeitada como base para uma decisão de não renovação de matrícula e que o encaminhamento para instituições de ensino especiais deve ser uma medida excepcional, e é proposta uma primazia da "inclusão" sobre a "integração".

\section{Palavras-chave}

Necessidades educacionais; discriminação educacional; infância; educação inclusiva; direito à educação.

\section{INTRODUCCIÓN}

Uno de los grandes desafíos que deben enfrentar las sociedades actuales y, dentro de ellas, los ordenamientos jurídicos, es la lucha en contra de las discriminaciones y las consecuentes violaciones de derechos. Existen grupos de personas particularmente vulnerables, entre otros, las mujeres, los inmigrantes (especialmente si no han regularizado su situación en su lugar de residencia), la comunidad $\mathrm{LGBTQ}^{+}$, las personas mayores, los que presentan alguna discapacidad y los niños, niñas y adolescentes (en adelante, NNA). Súmese a lo señalado, que es posible que una persona reúna más de uno de los nombrados factores de discriminación.

El presente trabajo se inspira en la consideración de la situación de un colectivo que debe afrontar con frecuencia diversas situaciones de discriminación. Con ello hacemos alusión a los NNA que tienen un trastorno del espectro autista (en adelante, TEA). Lo señalado se justifica porque es evidente que para este respecto deben adoptarse las medidas necesarias para librarlos de tales discriminaciones y, en consecuencia, contribuir al logro de su interés superior. En dicho sentido, una cuestión fundamental está dada por el acceso a la educación y el correspondiente avance curricular, dado que una debida formación educacional resulta ser clave para un adecuado desarrollo de todo NNA.

Teniendo en vista lo recientemente indicado, este artículo se centra en el análisis de la situación de los NNA que tienen necesidades educativas especiales (en adelante, NEE). En concreto, nos referiremos a los NNA que presentan algún tipo de TEA, a la luz del principio del interés superior del NNA. A propósito de dicho principio, junto con la normativa chilena que garantiza el ejercicio del derecho de la educación libre de discriminaciones, trataremos el iter educativo de un NNA con TEA. En conformidad con lo señalado, distinguiremos, por una parte, entre el proceso de admisión y la importancia de los Programas de Integración Escolar y, por la otra, la eventual derivación a un establecimiento especializado 
de educación. Así, destacaremos el deber de los recintos públicos o que reciben aportes del Estado, de admitir sin discriminaciones a NNA que tienen un TEA, la relevancia y utilidad de los Programas de Integración Escolar y la falta de entidad de la mera existencia de un TEA como fundamento de una decisión de no renovación de matrícula. Además, nos referiremos al dilema planteado por la falta de avances en el proceso de enseñanza-aprendizaje. Y nos pronunciaremos a favor del carácter de ultima ratio que debe tener la sanción de expulsión.

Finalizaremos este artículo propugnando la necesidad de transitar desde una etapa focalizada en la integración hacia un estadio en el que prime la inclusión de los NNA que presentan un TEA.

Con respecto a la metodología, se empleará el método dogmático, que utiliza, de modo principal, el análisis de documentos a partir de la revisión de la doctrina, jurisprudencia y normas nacionales e internacionales en materia de NEE, inclusión e interés superior. A mayor detalle, con respecto a la legislación chilena empleada, este trabajo se basa, fundamentalmente, en la disciplina de la educación en Chile (Ley $N^{\circ}$. 20.370) y, de un modo más específico, en el tratamiento de la subvención estatal a los recintos educacionales (Decreto con Fuerza de Ley $\mathrm{N}^{\circ} .2$ de 1996) y de la igualdad de oportunidades e inclusión social de personas con discapacidad (Ley $N^{\circ}$. 20.422). Desde el punto de vista del Derecho Internacional, resulta particularmente relevante el análisis del trabajo del Comité de los Derechos del Niño (observaciones generales $\mathrm{n}^{\circ}$. 1, 5, 9 y 14).

Con respecto a la jurisprudencia, se ha estudiado la emanada en la última década de los tribunales superiores de justicia chilenos, esto es, la Corte Suprema y las Cortes de Apelaciones. Dicha jurisprudencia ha permitido la aplicación de un método empírico que ha posibilitado, sobre la base de casos particulares, extraer conclusiones que poseen un carácter general basadas en los principios asumidos por los mencionados tribunales.

Sin perjuicio de lo recientemente indicado, a la luz del contenido de la Convención de los Derechos del Niño (especialmente su artículo 2, que regula la no discriminación arbitraria; artículo 3, precepto que consagra el principio del interés superior del NNA; y artículos 28 y 29, ambos relativos al derecho a la educación) y de la Convención de los Derechos de las Personas con Discapacidad (sobre todo, su artículo 7 titulado "Niños y niñas con discapacidad"), entre otros textos, es decir, sobre la base de la búsqueda del mejor interés del NNA y de la eliminación de toda forma de discriminación arbitraria, consideramos que la presente investigación plantea dilemas y propone conclusiones aplicables no solamente a la realidad chilena.

En otros términos, como diremos en las próximas páginas, el TEA no debe considerarse un obstáculo insalvable llegado el momento de aplicar en favor de los NNA de cualquier país las exigencias derivadas del derecho a la educación, lo que implica el acceso a ella, el correspondiente avance curricular y la no derivación, salvo supuestos muy excepcionales, a recintos de educación especial. De este modo, sobre la base del principio del interés superior y de la no discriminación arbitraria, es perfectamente viable construir espacios educacionales 
inclusivos que contribuyan de un modo efectivo al desarrollo de los NNA, con independencia del lugar geográfico que habiten.

\section{LA PARTICULAR SITUACIÓN EDUCATIVA DE LOS NNA CONTEA}

Como punto de partida, resulta pertinente tener presente que la educación es un key right, es decir, tal como lo ha indicado el Comité de Derechos Económicos, Sociales y Culturales (1999, p. 10) de la ONU, el derecho a la educación permite ejercer otros derechos humanos, de este modo, es el "epítome de la indivisibilidad y la interdependencia de todos los seres humanos". Si lo anterior es relevante para los NNA que no tienen una NEE, la noción del derecho a la educación como un key right adquiere una dimensión, incluso, más trascendente con respecto al interés superior de los NNA que presentan un TEA (FLORES, 2014, p. 109-122).

La doctrina está constituida con el fin de sostener que el principio del interés superior del NNA debe ser entendido como una noción amplia. En efecto, siguiendo a Ravetllat y Pinochet (2015, p. 916-919), el legislador poco incide en el contenido particular de dicho principio, el que se configura como un concepto jurídico indeterminado, con remisión, para su delimitación efectiva, al tiempo y a la persona que deba aplicar el precepto correspondiente $y$, por lo tanto, el concepto, con la necesaria adecuación del mandato legal a cada caso determinado, a la diversa variedad de personas implicadas y a las situaciones que pudieran generarse. Según López-Contreras (2015, p. 55), el principio en comento hace referencia al bienestar de los NNA, y basándose en el artículo 3 de la Convención Internacional sobre los Derechos del Niño (en adelante, CDN), debe prevalecer sobre toda otra "circunstancia paralela por la cual se tenga que decidir". Añade el nombrado autor que se debe tener en cuenta aquello más conveniente para el NNA en el caso concreto, mediante determinaciones que así lo indiquen. Lo dicho, puesto que, como es fácil apreciar, estamos en presencia de un concepto vinculado con las coordenadas personales y circunstancias del supuesto de que se trate y que evoluciona con el transcurso de los años. En similar sentido se ha pronunciado la doctrina especializada (RODRÍGUEZ, 2009, p. 568; LATHROP, 2005, p. 33; ROCA, 1994, p. 975; LOVERA, 2009, p. 215-220; MIRALLES, 1989, p. $97-$ 98, ORREGO, 2007, p. 14; GÓMEZ DE LA TORRE, 2000, p. 25-26). Por ello, el principio nos recuerda que las soluciones pertinentes jurídicas deben basarse tanto en la forma como en el contenido, en lo más beneficioso para los derechos de los niños sancionados legalmente (LEPÍN, 2013, p. 287).

En la misma línea de razonamiento, ¿cómo aplicamos lo anterior al objeto de nuestro estudio? Entendemos que, para efectos de construir el interés superior de los NNA con un TEA en el ámbito educacional, hay que considerar la particular vulnerabilidad que estos presentan (BARCIA, 2018, p. 481), la que se concreta en NEE, según analizaremos con mayor detalle en las líneas siguientes. 
Con respecto a la aludida situación de vulnerabilidad, para comprenderla adecuadamente debemos considerar las características propias del TEA. En dicho sentido, esta condición neurológica que se presenta desde la infancia influye, entre otros aspectos, en la manera en que las personas que la padecen se comportan, en su interacción social y en sus posibilidades de aprendizaje (ARTIGAS-PALLARÈS y PAULA, 2011, p. 572-574; ASTUDILLO y MONDACA, 2019, p. 262-263; BAÑA, 2015, p. 323; MARTÍNEZ y PIQUERAS, 2017, p. 200; BONILLA y CHASKEL, 2016, p. 5).

Sin perjuicio de lo expresado, debemos reflexionar acerca de la noción de vulnerabilidad, pues el hecho de que un NNA padezca de TEA no lo vuelve vulnerable. En efecto, la vulnerabilidad misma se genera ante la ausencia de un sistema de apoyos, esto es, ante la falta de recursos y estrategias que tienen por objeto promover el desarrollo, la educación, los intereses y el bienestar de una persona y mejorar, de esta manera, el funcionamiento individual. En lo anterior un relevante rol corresponde a los docentes, profesionales de la educación y a la familia, puesto que conforman los contextos en los que se desarrollan los NNA (ARAVENA, 2019 , p. 324). Por lo señalado, la carencia de tales apoyos genera una exposición a situaciones de vulnerabilidad. A propósito de este último aspecto, resaltamos la importancia de estudiar el TEA desde la perspectiva de las NEE, pues si el TEA impacta en el modo en que aprenden los NNA, corresponde asegurar el acceso a la educación de tales NNA, pero teniendo en vista sus características propias.

Considerando lo indicado, a propósito de las NEE de los NNA con un TEA, debe tenerse en cuenta, como lo ha expresado el Comité de los Derechos del Niño (2001, p. 7):

Que el objetivo principal de la educación es el desarrollo de la personalidad de cada niño, de sus dotes naturales y de su capacidad, reconociéndose el hecho de que cada niño tiene características, intereses y capacidades únicas y también necesidades de aprendizaje propias.

En esta senda, ha expresado Silveira (2016, p. 2) que es preciso tener en vista la búsqueda del mejor interés del NNA en cada una de las decisiones que se adopten respecto a las trayectorias educativas y particularmente en aquellas donde la complejidad que las atraviesa actúa como un impedimento para habilitar, alojar e incluir.

Para un mejor análisis del tema, a propósito del iter educativo de un NNA con un TEA, distinguiremos los siguientes aspectos: admisión y relevancia de los Programas de Integración Escolar y eventual derivación a un establecimiento especializado de educación.

\section{ADMISIÓN Y RELEVANCIA DE LOS PROGRAMAS DE INTEGRACIÓN ESCOLAR}

En una etapa tan crucial para la concreción del interés superior del NNA, como lo es la fase de admisión a un establecimiento de educación, adquiere una especial entidad la aplicación del principio de no discriminación. Como lo ha planteado Lovera (2015, p. 25), es: 
En el campo educacional donde se presenta la mayor cantidad de demandas por acomodos razonables para NNA con capacidades diversas. Aquí, los problemas no son solo de accesibilidad al espacio físico, sino que variados y complejos y que incluyen los procesos de admisión y selección.

Según el artículo 13 de la Ley General de Educación, en su texto modificado por la Ley Nº. 20.845, los procesos de selección de estudiantes deberán ser objetivos, transparentes, debidamente publicados, no pueden generar, en caso alguno, discriminaciones arbitrarias y deben garantizar el respeto a la dignidad de todos los alumnos, alumnas y sus familias, de acuerdo a las garantías consagradas tanto en la Constitución como en los tratados internacionales sobre derechos humanos suscritos y ratificados por Chile, "en especial aquellos que versen sobre derechos de los niños y que se encuentren vigentes" (CONGRESO NACIONAL DE CHILE, 2010a).

También debemos tener en vista lo dispuesto en el inciso $1^{\circ}$ del artículo 1 de la Ley $\mathrm{N}^{\circ}$. 20.422, en cuya virtud el Estado "garantizará a las personas con discapacidad el acceso a los establecimientos públicos y privados de educación regular o, en su caso, a los establecimientos que constituyen la educación especial, que reciban subvenciones o aportes del Estado" (CONGRESO NACIONAL DE CHILE, 2010b). Agrega el inciso $2^{\circ}$ de la citada norma que los recintos de enseñanza parvularia, básica y media contemplarán planes para alumnos y alumnas con NEE y, además, fomentarán la participación en estos de todo el plantel de académicos y académicas, asistentes de educación y demás miembros de la comunidad educacional.

Resulta claro que el principio de no discriminación debe impregnar los distintos criterios de admisión aplicados por los recintos educacionales (CORNEJO, 2019, p. 34-35; JUARROS, 2006, p. 69-71). Con todo, ante el hecho objetivo constituido por un NNA que presente un TEA, cuyos padres deseen matricularlo en un determinado colegio o escuela, surge la siguiente pregunta: ¿pueden estos últimos, sin incurrir en discriminaciones arbitrarias, negarse a los deseos paternos? Para responder adecuadamente la interrogante planteada, es necesario distinguir entre el sistema que recibe fondos públicos y el sistema privado de educación.

Con respecto a lo primero, los colegios y escuelas que reciben aportes del Estado, esto es, los establecimientos educacionales públicos y los reconocidos oficialmente por el Estado (ex subvencionados), no pueden rechazar el ingreso de un NNA que tenga un TEA. A mayor abundamiento, respecto de tales establecimientos, según el procedimiento de asignación de vacantes establecido en la Ley $\mathrm{N}^{\circ} .20 .845$ y en el Decreto $\mathrm{N}^{\circ} .152$ del Ministerio de Educación, aplicable desde el primer nivel de transición hasta cuarto año medio, tanto para recintos de enseñanza científico humanista como técnica profesional, quedan expresa-

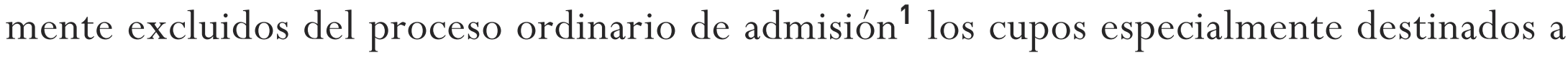

1 En síntesis, dicho proceso se estructura sobre la base de los siguientes aspectos: número de vacantes, aplicación de criterios de prioridad y de números otorgados de manera aleatoria. Sin perjuicio de lo anterior, 
estudiantes que presenten NEE y permanentes: tales estudiantes podrán ser incluidos en los denominados "Programas de Integración Escolar" (en adelante, PIE), existentes en el establecimiento escolar. Es pertinente resaltar que son los propios recintos educacionales los encargados de fijar un procedimiento de admisión a los PIE (HOLZ, 2019, p. 2). Todo lo indicado constituye un cumplimiento por parte del Estado de Chile tanto de las normas de su Derecho interno como de las normas de Derecho internacional que le obligan a no incurrir en discriminaciones arbitrarias y a considerar las NEE de los NNA.

Panorama diverso se aprecia en el sistema privado de educación. La libertad que el ordenamiento jurídico garantiza a los particulares les permite desarrollar una serie de actividades, entre ellas, la creación y gestión de recintos educacionales. En esta senda de razonamiento, es lícito que la autonomía de la voluntad de los particulares les conduzca a establecer una normativa que persiga el logro de altos estándares académicos. En dicho contexto, es viable que un NNA con un TEA no sea seleccionado en los procesos de admisión de los colegios y escuelas privadas. En otros términos, dada su naturaleza no pública, sumada a la ausencia de financiamiento estatal, el Estado no puede imponer a los recintos de educación privados la matrícula de NNA con NEE. Además, el Estado no puede delegar en entes privados la labor de otorgar una adecuada educación a los NNA que presenten un TEA. Sin perjuicio de lo anterior, el descrito estado de cosas es llamativo, dado que, los NNA que presenten un TEA podrán ser admitidos, en el contexto de la educación privada, solamente por aquellos recintos que contemplen planes especiales para satisfacer sus necesidades educativas. Dado que lo anterior no ocurre en todas las ciudades chilenas, en varias de estas, no existe opción alguna, es decir, tales NNA deberán estudiar necesariamente en recintos públicos.

Por todo lo indicado, en la etapa de admisión, la consideración de la existencia de un TEA podría justificar y, en consecuencia, eliminar la arbitrariedad de la decisión del recinto educacional privado relativa a no permitir el ingreso de un NNA. Lo anterior fue reconocido por la Corte de Apelaciones de Antofagasta (2018), al resolver en el considerando $6^{\circ}$ de la sentencia definitiva de la causa rol nº $3149-2018$ que: "cualquier negativa de matrícula o ingreso al establecimiento educacional debió efectuarse cuando se requirió el ingreso, puesto que era ésta y no otra, la oportunidad para evidenciar las supuestas falencias de un sistema de integración escolar". En suma, la naturaleza del recinto educacional y la fuente del financiamiento escolar podría contribuir a excluir la discriminación en la etapa de admisión.

También debemos tener presente que el Decreto Supremo No. 170 y el artículo 23 de la Ley General de Educación no obligan a los establecimientos educacionales públicos y a los

los planteles educacionales que persigan la excelencia académica, deportiva o artística, poseen mecanismos especiales de admisión. 
reconocidos oficialmente por el Estado a contar con un PIE. Lo anterior es un factor que debe ser tomado en cuenta, entre tantos otros, por los padres al momento de decidir acerca del colegio en el que estudiarán sus hijos, pues estos deben velar por el mejor interés de los NNA. Lamentablemente, dicho aspecto suele ser desconocido, en general, por nuestra sociedad y, de un modo más específico, por los padres. ${ }^{2} \mathrm{Si}$ el respectivo recinto educacional carece de un PIE, será misión, en esencia, de los padres, en virtud de su derecho-deber de crianza, cuidado y educación (acudiendo a los establecimientos de salud pública o privada u otros recintos), proveer a sus hijos e hijas que presentan un TEA de los servicios profesionales necesarios pertinentes (terapias y trabajos complementarios, entre otros aspectos) que permitan apoyarles en su proceso educativo.

No olvidemos que lo que se entenderá por interés superior dependerá de cada caso específico y debe precisarse de forma individual, considerando la situación concreta del NNA afectado y el entorno, la situación y las necesidades personales, así lo ha sostenido el Comité de los Derechos del Niño (2013) en la Observación $N^{\circ}$. 14. Por lo tanto, resulta claro que el contexto, la situación y necesidades particulares deben valorarse detenidamente si lo pretendido es que el hijo o hija logre el mayor bienestar posible y multiplique sus potencialidades, lo que estimamos se obtiene direccionando los procesos educativos hacia la "inclusión".

Lo anterior se logra de mejor manera mediante la regulación y la implementación de un PIE en los establecimientos educacionales. A mayor abundamiento, el Ministerio de Educación, en las orientaciones para los sostenedores, sugiere aplicar los PIE, dado que permiten poner a disposición del sistema escolar recursos humanos y materiales adicionales para proporcionar apoyos a estudiantes que presentan NEE en los establecimientos de educación regular y, de este modo, equiparar oportunidades de participación y progreso en su aprendizaje. ${ }^{3}$ Estos programas generalmente cuentan con personal especializado, psicólogos, educadoras diferenciales, fonoaudiólogos y/o terapeutas ocupacionales, entre otros profesionales. Los PIE son financiados por medios de un sistema de subvenciones, ello en conformidad a lo establecido en el Decreto Supremo $N^{\circ}$. 170, normativa que disciplina el funcionamiento y los requisitos de estos programas. Las acciones que realizan los PIE se

2 El Decreto Supremo No 170 es un reglamento que regula las NEE y la subvención escolar que reciben los recintos educacionales por cada alumno o alumna que tiene una NEE. Para recibir la subvención estatal, el respectivo establecimiento debe contar con un PIE. Por lo anterior, es voluntario para los colegios y escuelas la existencia de un PIE, en otros términos, si el establecimiento no solicita la subvención especial, no se verá obligado a implementar un PIE, tal como se disciplina en los artículos 83 y siguientes del Decreto Supremo 170.

3 Antecedentes generales de PIE. MINISTERIO DE EDUCACIÓN. Ayuda Mineduc atención ciudadana. Disponibles en: https: / / www.ayudamineduc.cl/ficha/antecedentes-generales-pie-5. Acceso en: 16 de mayo de 
enmarcan en el diagnóstico, que suele ser interdisciplinario e integral y con inclusión de la familia, y en la planificación de acciones específicas atendiendo a la diversas necesidades educativas. Tales acciones se concretan en un plan de intervención individual para cada NNA, así, a propósito de los alumnos y alumnas con un TEA, necesariamente se requiere la intervención del psicólogo.

Una prueba de la contribución realizada por los PIE a una real inclusión, está dada por la forma común en que tales programas se concretan en las aulas de las unidades educativas. En efecto, en las salas de clases encontramos juntos a los NNA con alguna NEE con los demás estudiantes, lo último, sin perjuicio de intervenciones individuales de los profesionales anteriormente nombrados. Excepcionalmente, cuando el caso lo amerite, los estudiantes con una NEE son reunidos en un curso paralelo.

En definitiva, en virtud de la existencia de los PIE, los NNA de los sectores económicos más vulnerables de nuestra sociedad han podido acceder a una atención profesional especializada, pues los PIE están constituidos en la mayoría de los establecimientos educacionales municipales y ex subvencionados. De este modo, los programas nombrados se han constituido en un vehículo que ayuda a democratizar el acceso al apoyo académico y profesional especializado en el tratamiento de las NEE, promoviéndose así la igualdad de oportunidades entre los NNA.

No obstante lo expresado, es importarte ilustrar al lector en el siguiente sentido: el sistema educacional chileno, desde el año 1981, ha sido financiado mediante subvenciones, lo que implica que los establecimientos educacionales reciban financiamiento por cada alumno matriculado, por lo tanto, el mayor o menor número de recursos financieros es proporcional al número de estudiantes que tenga cada escuela o colegio. Se debe tener presente que el hecho de estar los PIE solventados bajo esta lógica responden a "una perspectiva política y económica específica, donde la descentralización y la competencia toman un lugar preponderante" (NÚÑ̃Z et al., 2020, p. 349), pues los recintos educativos postulan a estos fondos, y en la medida que dentro de su matrícula existan estudiantes con un diganóstico de necesidad educativa, obtendrán una subvención aumentada.

Aplicar a los PIE el modelo de subvención escolar devela la intención del legislador chileno de "consolidar el sistema de mercado sin querer insistir en las dificultades de carácter

2021. Podemos resumir las etapas de postulación a los PIE del siguiente modo: labor de sensibilización dirigida a los apoderados, relativa a la necesidad de contar con este programa; determinar el número de estudiantes que presentan NEE; aplicar diagnósticos integrales a estudiantes que se encuentren en la situación descrita; el sostenedor debe postular a través de los mecanismos que dispone el MINEDUC, y debe acreditar que cuenta con el apoyo necesario de profesionales idóneos; una vez que es aceptada la postulación, debe presentarse ante los organismos correspondientes la propuesta de PIE. 
político que atañen a su funcionamiento" (PEÑA, 2013, p. 96). Otro aspecto criticable es el que sigue: el Decreto $\mathrm{N}^{\circ}$. 170 tiene como principal herramienta de gestión el diagnóstico, sin profundizar en cómo se implementan y funcionan los PIE. En atención a lo indicado, es necesario considerar, además, la debida fiscalización del modo en que se desarrollan los PIE.

\section{EVENTUAL DERIVACIÓN A UN ESTABLECIMIENTO ESPECIALIZADO DE EDUCACIÓN}

En esta parte, como punto de partida, es necesario afirmar lo siguiente: por regla general, lo más beneficioso para el interés superior del NNA es la permanencia de estos en los recintos de educación en los que hubiesen sido admitidos. Por lo tanto, la no renovación de la matrícula respectiva (o la expulsión), de la que se deriva el traslado a un nuevo colegio o escuela, debe ser una medida excepcional, esto es, debe ser la ultima ratio y, en consecuencia, deberá dicha medida ser suficientemente fundada sobre la base del propio mejor interés del NNA de que se trate, en el contexto de un debido proceso.

Lo recientemente señalado exige que la sanción de expulsión sea notificada a los padres o representantes legales, y que el respectivo reglamento de convivencia contemple mecanismos de descargos o de revisión de las sanciones. Lo anterior es una manifestación del hecho de considerar a los NNA como sujetos de derecho, pues tienen derecho a que en cualquier procedimiento disciplinario se respeten las garantías básicas de un proceso previo, racional y justo. Además, como lo ha resuelto la Corte de Apelaciones de San Miguel (2020) en la sentencia dictada en la causal rol n ${ }^{\circ}$ 3936-2020, la sanción aplicada debe ser proporcional a la falta cometida, en este contexto el principio de proporcionalidad:

Funciona como un mecanismo de control a la discrecionalidad de la autoridad educativa, que requiere una relación directa entre la gravedad del acto cometido y la sanción que lleva aparejada, no siendo pertinente entonces, que se aplique la medida de expulsión o cancelación de matrícula respecto de hechos que no atenten gravemente contra la convivencia escolar.

De no cumplirse con lo indicado, la decisión del ente educacional deviene en arbitraria e, inclusive, en ilegal. A mayor detalle, según lo dispuesto en letra f) del artículo 46 del Decreto con Fuerza de Ley $N^{\circ}$. 2, del 2 de julio de 2010, precepto que enumera los requisitos que deben cumplir los establecimientos que imparten educación parvularia, básica y media para tener reconocimiento del Ministerio de Educación, tales establecimientos deben contar con un reglamento interno que incorpore normas relativas a la buena convivencia escolar. Con respecto a lo último, pueden establecerse en el mencionado texto diversas medidas disciplinarias, siendo la más gravosa de ellas la expulsión, siempre que sea el resultado de un debido proceso, según ya hemos indicado. Así, los supuestos que hacen 
procedente la máxima sanción que puede recaer sobre un alumno o alumna, esto es, la no renovación de su matrícula, deben encontrase explícitamente previstos en la normativa señalada (CONGRESO NACIONAL DE CHILE, 2010b).

Por lo señalado en los párrafos precedentes, resulta relevante indicar los casos en que la derivación del NNA a un nuevo colegio o escuela es recomendable desde el punto de vista del interés superior y no constituye un atentado a su interés superior. Profundizando en esta idea, analizaremos las siguientes cuestiones: la insuficiencia de un TEA para fundamentar por sí la decisión de no renovación de matrícula y la falta de avances en el proceso de enseñanza-aprendizaje.

\section{LA INSUFICIENCIA DE UNTEA PARA FUNDAMENTAR POR Sí LA DECISIÓN DE NO RENOVACIÓN DE MATRÍCULA}

De ningún modo la expulsión puede tener como fundamento la mera existencia de una NEE, pues ello es contrario a derecho, ya que el artículo 2 de la Ley $\mathrm{N}^{\circ}$. 20.845 modificó el artículo 6 de la Ley de Subvenciones, prescribiendo expresamente lo siguiente:

Los sostenedores y, o directores no podrán cancelar la matrícula, expulsar o suspender a sus estudiantes por causales que se deriven de su situación socioeconómica o del rendimiento académico, o vinculadas a la presencia de necesidades educativas especiales de carácter permanente y transitorio definidas en el inciso segundo del artículo $9^{\circ}$, que se presenten durante sus estudios. A su vez, no podrán, ni directa ni indirectamente, ejercer cualquier forma de presión dirigida a los estudiantes que presenten dificultades de aprendizaje, o a sus padres, madres o apoderados, tendientes a que opten por otro establecimiento en razón de dichas dificultades. (CONGRESO NACIONAL DE CHILE, 1998)

Lo indicado se vincula con el contenido de los artículos 34 y 36 de Ley $\mathrm{N}^{\circ}$. 20.422. En efecto, según lo prescrito en el primer artículo mencionado, los establecimientos de educación parvularia, básica y media deberán contemplar planes para alumnos con NEE y fomentarán en ellos la participación de los profesores y profesoras, asistentes de educación y demás integrantes de la comunidad educacional. Por su parte, según el artículo 36, los colegios y escuelas de enseñanza regular deberán introducir las innovaciones y adecuaciones curriculares, de infraestructura y los materiales de apoyo necesarios para permitir y facilitar a las personas con discapacidad el acceso a los cursos o niveles existentes, otorgándoles los recursos adicionales que fuesen necesarios para asegurar su permanencia y progreso en el sistema educacional (CONGRESO NACIONAL DE CHILE, 2010a).

Como podemos advertir, los recintos educacionales, según los citados artículos, deberán necesariamente implementar los ajustes necesarios en sus planes de desarrollo de forma 
tal de atender de manera adecuada a los estudiantes que presenten algún tipo de NEE, tanto desde un criterio pedagógico como técnico.

A mayor abundamiento, la letra a) del artículo 10 de la Ley $\mathrm{N}^{\mathrm{o}}$. 20.370, prescribe que los establecimientos educacionales deben prestar una atención que se caracterice por ser adecuada y oportuna y, en el caso de estudiantes con necesidades especiales, prescribe que estos no deben ser discriminados arbitrariamente. Súmese que, en conformidad a lo indicado en el inciso $1^{\circ}$ del artículo 23 de la mencionada legislación, los establecimientos regulares deben proveer un conjunto de servicios, recursos humanos, técnicos, conocimientos especializados y ayudas que tengan por fin satisfacer las NEE de los NNA a lo largo de todo el proceso de enseñanza (CONGRESO NACIONAL DE CHILE, 2010b).

Volviendo a lo dispuesto en los artículos 34 y 36 de la Ley $N^{\circ}$. 20.422, en estas normas se establecen deberes de actuación para los colegios y escuelas vinculados al modo de proceder respecto de los estudiantes con NEE. Así, se regulan tres deberes, dos en el artículo 34 y uno en el artículo 36. En el artículo 34 se disciplinan los siguientes deberes: en primer lugar, elaborar planes para alumnos con NEE y, en segundo término, fomentar la participación activa en tales planes del plantel de profesores, asistentes de educación y, en términos amplios, de los demás miembros de la comunidad educacional. Estos dos deberes apuntan a la preparación que debe implementar los colegios y escuelas con vistas a admitir y posibilitar la continuidad del proceso de enseñanza-aprendizaje respecto de NNA con NEE.

El tercer deber se ha disciplinado en el artículo 36, en su virtud, los recintos educacionales deben introducir las innovaciones y adecuaciones pertinentes en sede curricular y en lo relativo a su infraestructura. Además, deberán proporcionar los materiales de apoyo adecuados para "permitir" y "facilitar" a las personas con necesidades especiales el acceso a los cursos o niveles existentes. Para cumplir con lo señalado, será menester disponer de los recursos adicionales necesarios para garantizar tanto la permanencia como el progreso en el sistema educacional. En otras palabras, no basta con hacer viable la matrícula de un NNA que presente un TEA, sino que, además, los recintos educacionales deben colaborar activamente en el logro de lo anterior.

Por todo lo dicho, resulta claro que los recintos educacionales que reciben fondos estatales no pueden decidir la no renovación de una matrícula en razón de la presencia en un NNA de un TEA. Con respecto a los establecimientos privados, si un NNA con un TEA ya ha sido admitido, no es correcto que, sobre la base del mismo TEA, se intente con posterioridad justificar la no renovación de su matrícula. Así, al aceptarse la matrícula de un NNA con un TEA, el recinto educacional asume la responsabilidad de brindarle los pertinentes servicios de educación. Tanto respecto de los establecimientos públicos como a propósito de los recintos privados, los últimos en el contexto indicado, la no renovación de matrícula constituye un acto discriminatorio que, a todas luces, atenta en contra del principio del interés superior del NNA.

En efecto, corresponde al colegio o escuela adoptar las medidas necesarias tendientes a satisfacer las NEE de un estudiante con un TEA. Dicho con otras palabras, no es aceptable que 
un recinto educacional, acudiendo para ello a una expulsión, pretenda desentenderse de los deberes propios de la labor de educar a un NNA que presente las características indicadas.

Este criterio ha sido sostenido por la Corte de Apelaciones de Antofagasta (2018) en la causa rol $n^{\circ}$. 3149-2018, al establecer en el considerando $6^{\circ}$ de la sentencia recaída en dicha causa lo siguiente (el establecimiento recurrido formaba parte de la educación privada):

El recurrido conocía desde el ingreso del niño a su establecimiento educacional que este presentaba espectro de autismo, lo que implicaba e implica necesidades que requerían de una atención especial y/o distinta respecto de sus otros educandos, pese a lo cual lo aceptó e incorporó a su proyecto educativo en el año 2016 según la recurrente, desde 2015 según el recurrido.

Agrega la sentencia individualizada que:

Sea uno u otro, hace por lo menos, tres años lectivos, de modo tal que habiéndolo incorporado a su proyecto educacional con conocimiento de su particular situación, todas las argumentaciones para no renovar o para cancelar su matrícula en la actualidad devienen en arbitrarias o caprichosas porque cualquier negativa de matrícula o ingreso al establecimiento educacional debió efectuarse cuando se requirió el ingreso, puesto que era esta y no otra, la oportunidad para hacer presentes las supuestas falencias de un sistema de integración escolar.

En consecuencia, en virtud de todo lo señalado, se reafirma que la mera existencia de un TEA no puede emplearse como basamento de una decisión de expulsión, por el contrario, es misión de los establecimientos educacionales prepararse debidamente para recibir a tales estudiantes y apoyarles durante todo su proceso académico.

Con todo, en ocasiones, la realidad demuestra que es posible que, no obstante la aplicación de un PIE, lo más beneficioso para el interés de un NNA sea retirarse del procedimiento ordinario de enseñanza. En tales casos, surge la opción de clases especiales impartidas en el mismo colegio o escuela, o en otro establecimiento educacional especializado. ${ }^{4}$ En dicho sentido, en conformidad a lo regulado en el inciso $2^{\circ}$ del artículo 36 de la Ley $\mathrm{N}^{\circ}$. 20.422,

4 La Ley $\mathrm{N}^{\circ}$. 20.422, que establece normas sobre igualdad de oportunidades e inclusión social de personas con discapacidad, dispone en su artículo 35 que la educación especial es una modalidad del sistema escolar que otorga servicios y recursos especializados, con el objetivo de asegurar aprendizajes de calidad a NNA con NEE asociadas o no a una discapacidad, de forma tal de cumplir con el principio de igualdad de oportunidades respecto de todos los educandos (CONGRESO NACIONAL DE CHILE, 2010a). 
si la integración en los cursos de enseñanza regular no es posible, considerando la naturaleza y tipo de la discapacidad del estudiante, la enseñanza deberá impartirse en clases especiales dentro del mismo establecimiento educacional o en recintos especiales. Lo recientemente afirmado nos conduce a razonar sobre los efectos de la falta de avances curriculares de los NNA con NEE, aspecto que trataremos con más detalle en las líneas siguientes.

\section{LA FALTA DE AVANCES EN EL PROCESO DE ENSEÑANZA-APRENDIZAJE}

Un punto de ineludible estudio está referido al nivel de éxito obtenido en el proceso de enseñanza-aprendizaje. En efecto, dadas las características propias del TEA, es viable que algunos de los NNA que tienen algún grado de dicho trastorno no puedan lograr el mismo nivel de avance curricular obtenido por los estudiantes que no presentan un TEA, a pesar de los esfuerzos realizados por el plantel educacional respectivo. Una verdadera preocupación por el interés superior de los NNA con un TEA exige que, ante la falta de avances en el proceso de enseñanza-aprendizaje, se reevalúen las estrategias pedagógicas hasta el momento aplicadas y, eventualmente, sobre la base de dicho análisis, se decida implementar algún tipo de innovación académica o, como ultima ratio, la derivación a un establecimiento especializado.

Es necesario subrayar la última idea, dado que la realidad demuestra que algunos recintos educacionales, en ocasiones, entienden que el mal rendimiento les habilita para expulsar a un NNA con un TEA. En una acción de protección resuelta por la Corte de Apelaciones de Antofagasta (2018), en la ya citada causa rol nº.3149-2018, en términos explícitos, tal como se indica en el considerando $6^{\circ}$ de la sentencia, el recurrido indicó que un negativo rendimiento académico justificaría su proceder:

La decisión (no) se ha sustentado en alguna medida disciplinaria producto de alguna transgresión al Manual o Reglamento de Convivencia, por el contrario, se sustenta en la situación de aprendizaje del menor en la educacion parvularia, que no ha presentado avances desde su ingreso, velando por no atentar contra su derecho a acceder a una educación especializada de acuerdo al diagnóstico que presenta.

En este caso el tribunal de alzada estimó que el escaso avance académico que pudo presentar el estudiante no era motivo suficiente para la expulsión. A mayor abundamiento, los sentenciadores concluyeron que la expulsión no obedecía a la configuración de una conducta sancionada en el Manual de Convivencia. Insistimos, no puede justificarse la expulsión de un NNA que presente un TEA en una mera relación de causalidad naturalística entre el negativo desempeño curricular (causa) y la sanción de expulsión (efecto).

También existen sentencias que han reconocido que, en casos excepcionales, lo mejor para el interés del NNA es su derivación a un recinto especializado, ello ante la acreditada imposibilidad de avances curriculares en los establecimientos educacionales que les habían 
admitido. En esta línea de razonamiento, la Corte de Apelaciones de Santiago (2015), en el juicio rol $n^{\circ}$. 82.836-2014, a propósito de una acción de protección deducida por una apoderada en representación de su hija expulsada, que tenía un trastorno de lenguaje, resolvió en el considerando $8^{\circ}$ de la sentencia definitiva:

Que así las cosas la decisión de la recurrida aparece racional y apoyada en motivaciones suficientes que resultan idóneas y fundada en la normativa legal señalada, que en todo caso resulta ser protectora de los derechos de la niña E., que requiere de apoyo y ayuda adicional en su proceso educativo; dado que se encuentra acreditado que ella presenta necesidades educativas especiales; todo ello sin perjuicio que la presente acción ha perdido oportunidad toda vez que la menor ya está asistiendo a un establecimiento educacional; por todo lo cual el recurso no podrá prosperar.

Similar sendero recorrió la Corte de Apelaciones de Valdivia (2015) en la sentencia pronunciada en causa rol $n^{\circ}$. 86-2015, órgano jurisdiccional que en el considerando $15^{\circ}$ de dicha resolución estableció que la decisión de cancelación de matrícula de un niño que tenía un trastorno generalizado del desarrollo no era ni arbitraria ni ilegal:

Se ha podido observar que se han brindado las herramientas pertinentes para cubrir las necesidades especiales del menor, sin que ello haya significado una evolución en su proceso de desarrollo educativo y social, como se refleja de las constancias existentes en este proceso de las entrevistas efectuadas y los informes elaborados por los profesionales del establecimiento recurrido, rolantes a fojas 7 a 16 y de fojas 180 a 206, los que apreciados según las normas de la sana crítica, no logran establecer que la recurrida haya actuado en desprecio del derecho a la igualdad, consagrado en el artículo $19 \mathrm{~N}^{\circ} 2$ de la Constitución Política de la República o cometido actos discriminatorios.

A nuestro juicio, resulta correcto lo resuelto en las dos últimas mencionadas sentencias, además de los argumentos ya explicitados, por la naturaleza misma del TEA. En efecto, en términos generales, es pertinente recordar que el TEA es un trastorno que afecta el neurodesarrollo y, como hemos indicado previamente, está caracterizado por provocar una serie de dificultades en la comunicación y en la interacción social. ${ }^{\mathbf{5}}$

$5 \quad$ ElTEA se caracteriza por deficiencias persistentes en la comunicación y en la interacción social en distintos contextos. Lo señalado, se concreta en varias insuficiencias: en la reciprocidad socioemocional; en las conductas comunicativas no verbales utilizadas en la interacción social; y en el desarrollo, mantenimiento y comprensión de las relaciones. Súmese a lo señalado la existencia de patrones restrictivos y repetitivos 
El TEA constituye una “condición” que implica limitaciones en el desarrollo integral de una persona, prueba de lo anterior son las dificultades que plantea tanto a la incorporación regular de una persona a la sociedad en forma autónoma como a su relacionamiento interpersonal. Por todo lo indicado, los NNA que tienen un TEA requieren de un apoyo especializado y multisectorial, a lo que debe añadirse, en el plano familiar, la existencia de un ambiente que se caracterice por ser comprometido, responsable y dedicado (tal como se resolvió en la sentencia de la Corte Suprema, en causa rol no. 29566-2014 de 2015). En este sentido se pronunció la Corte Suprema de Chile (2019a), en el considerando $5^{\circ}$ de la sentencia definitiva de la causa rol no ${ }^{\circ}$ 1550-2019, al tener en vista lo indicado por la consejera técnico del Centro de Medidas Cautelares de Santiago:

En el caso de los niños con trastorno del espectro autista moderado las habilidades pre-académicas se desarrollan lentamente, evidenciándose notablemente reducidas en comparación de sus iguales, es así como el lenguaje es menos complejo que el de otros niños de su edad. Sin embargo, el desempeño en la capacidad de relación se vincula con su formación familiar y su entorno.

Con todo, en este escenario, sería poco acertado solo ponderar la variable estrictamente académica. En efecto, el TEA afecta el proceso de aprendizaje, además del entorno del NNA y su interacción social, aspectos todos que repercuten en el rendimiento. En este sentido desarrolló su argumentación la Corte Suprema conociendo de un recurso de apelación que impugnó una sentencia dictada por la Corte de Apelaciones de Arica, la que rechazó un recurso de protección presentado por la apoderada de un niño de siete años, quien solicitó al establecimiento educacional que recibía subvención estatal, por contar con un PIE, que se contratase un tutor destinado a brindar apoyo académico a su hijo.

A mayor detalle, la Corte de Apelaciones de Arica (2019), en la sentencia pronunciada en la causa rol $n^{\circ}$. 506-2019, rechazó la acción constitucional indicando en el considerando $8^{\circ}$ que:

El colegio recurrido, que recibe la subvención especializada, a juicio de esta Corte, ha cumplido las obligaciones que la misma le impone, sin que le sea obligatorio contratar

de comportamiento, intereses y actividades. Todo lo dicho puede apreciarse en las primeras fases de desarrollo del TEA de que se trate. ASOCIACIÓN AMERICANA DE PSIQUIATRÍA. Guía de Consulta de los Criterios Diagnósticos del DSM-5, 2013. Disponible en: http://www.eafit.edu.co/ninos/reddelaspreguntas / Documents/dsm-v-guia-consulta-manual-diagnostico-estadistico-trastornos-mentales.pdf. Acceso en: 16 de mayo de 2021. 
el tutor que reclama la madre del niño, lo que obliga a desestimar la presente acción constitucional.

Es decir, los sentenciadores interpretaron que el objetivo de la subvención especial otorgada por el Estado, regulada en el Decreto Supremo N ${ }^{\circ}$. 170, se cumple con la mera contratación y ejercicio de funciones de los profesionales del PIE.

Lo resuelto fue corregido por la Corte Suprema, tribunal que razonó lúcidamente sobre la base del contenido esencial delTEA, es decir, las dificultades en la interacción social y en el aprendizaje, por lo tanto, estableció nuestro máximo sentenciador que la labor de los establecimientos educacionales que perciben recursos del Estado no se puede limitar de manera exclusiva al apoyo estrictamente académico. Concordamos con lo resuelto, de esta manera, estimamos que no sería suficiente apreciar exclusivamente el avance académico de un NNA con un TEA, prescindiendo de otras variables, que permiten su verdadera inclusión, como motivo que habilite a los establecimientos educacionales a aplicar sanciones o expulsiones.

La Corte Suprema de Chile (2019b), en la sentencia recaída en la causa rol n ${ }^{\circ}$. 18.8102019 , en el considerando $6^{\circ}$, orientó su razonamiento en el sentido indicado, y a propósito de la apelación de la acción constitucional en comento, señaló:

Ha quedado asentado que el colegio estima que ha cumplido con lo dispuesto en la norma legal citada en el considerando quinto porque el alumno tiene un desempeño medio y al contar en aula con una profesora y una técnico auxiliar, sin embargo, estas acciones resultan insuficientes al tenor de la disposición legal referida, porque lo requerido es apoyo en la sala de clases, el que no puede restringirse solo a lo académico, toda vez que por el diagnóstico del niño, éste presenta dificultades en su interacción social. En este orden de ideas, que el desempeño del niño sea promedio es solo el resultado, pero no demuestra necesariamente que el mismo sea el resultado de un trabajo de planificación previo, en el que se haya involucrado al estudiante, a su familia y el entorno extendido de este.

En definitiva, solo de un modo excepcional el progreso académico (o la falta de éste), en el caso de los alumnos con NEE, puede justificar la derivación a un recinto especializado, pues, según hemos indicado, la labor de los recintos educacionales no se agota solo en la organización e implementación de recursos estrictamente académicos, sino que también debe considerar los aspectos familiares y, en términos más amplios, sociales.

Entender la derivación a un recinto especializado como medida de ultima ratio se fundamenta, también, en atención a las exigencias derivadas del interés superior. En efecto, se vulnera el interés superior del NNA si la falta de progreso académico es empleada como justificación automática de una decisión de no renovación de matrícula: primero debe intentarse 
que el NNA permanezca en su colegio y escuela. En esta senda, es sabido que el interés superior exige evitar los cambios que puedan perjudicar al NNA y entendemos que separarle del ambiente al que se ha acostumbrado le afecta de un modo negativo, ello porque implica, principalmente, el alejamiento de sus compañeros de clases y de los profesores e impide la continuidad de las dinámicas propias a las que se ha habituado, cuestión de suma importancia a propósito del TEA (HERVÁS y RUEDA, 2018, p. 534).

En suma, la derivación en comento puede aumentar las consecuencias negativas derivadas delTEA ya indicadas en el presente trabajo. Para demostrar lo anterior, basta con pensar en la dificultad que tales NNA tienen para adaptarse a los nuevos escenarios, de este modo, el cambio de colegio puede tener como efecto un aumento de la sintomatología y de las características del TEA. ${ }^{6}$

\section{REFLEXIONES FINALES: INTEGRACIÓN-INCLUSIÓN}

Es fundamental precisar que para el logro de un adecuado desarrollo de los NNA que tienen un TEA, resulta determinante orientar el proceso de enseñanza-aprendizaje hacia la “integración” y, especialmente, centrarlo en la "inclusión”, entendiendo que no estamos en presencia de expresiones sinónimas y, en consecuencia, poseen un sentido y alcance distinto. En efecto, Molina ha precisado que la integración se focaliza en un apoyo educativo a los alumnos y alumnas con NEE, para lo anterior se desarrollan innovaciones de apoyos, y se cuenta con recursos y profesionales capacitados en el ámbito de la educación especial. En cambio, la inclusión implica una mirada más omnicomprensiva, dado que se basa en un modelo que exige que la comunidad educativa y escolar en su conjunto esté preparada para admitir la diversidad existente mediante un proceso socio-comunitario (MOLINA, 2015, p. 151).

En otros términos, la inclusión tiene un campo de aplicación mucho más amplio, pues se vincula con el acceso, la participación y logros de todos los NNA, con un particular énfasis en los que se encuentran en situación de riesgo de ser excluidos o marginados (BLANCO, 2006, p. 6). Básicamente, siguiendo lo expresado por el Comité de los Derechos del Niño en la Observación General N.9 (2006, p. 21), la educación inclusiva es un conjunto

6 El MINEDUC ha afirmado que, en diversas investigaciones de carácter mundial, se ha encontrado que algunos de los aspectos que más favorecen la educación de los NNA con un TEA son: crear un ambiente estructurado y predecible; promover experiencias de aprendizaje significativo; y mantener contextos y objetivos individualizados de enseñanza. MINISTERIO DE EDUCACIÓN DE CHILE. Guía de apoyo técnico-pedagógico: Necesidades educativas especiales en el nivel de educación parvularia, p. 25, 2008. Disponible en: https://especial.mineduc.cl/wp-content/uploads/sites/31/2016/08/GuiaAutismo.pdf. Acceso en: 16 de mayo de 2021. 
de valores, principios y prácticas que tratan de lograr una educación cabal, eficaz y de calidad para todos los estudiantes, que hace justicia a la diversidad de las condiciones de aprendizaje y a las necesidades no solamente de los estudiantes con discapacidad, sino de todos los alumnos y alumnas. Por su parte, la integración se dirige al estudiante, a sus capacidades y necesidades, y apunta a la disposición de recursos humanos y materiales para la satisfacción de éstas. En suma, la inclusión apunta a poner la educación regular a disposición de todos los estudiantes tengan o no necesidades particulares.

En este punto, resulta pertinente volver a razonar sobre la existencia de los PIE. Así, aunque su propia denominación los califique como programas de integración, pueden constituirse en una estrategia que conduzca a una efectiva inclusión. En efecto, según hemos indicado precedentemente, en principio, los PIE apuntan a reunir en una misma aula a todos los estudiantes, tengan o no estos una NEE, brindando, en su caso, los apoyos que resulten necesarios. Con todo, si pese a los esfuerzos que se realicen por lograr una verdadera inclusión, estamos en presencia de estudiantes que tienen una NEE permanente que impide el referido trabajo mancomunado, como puede suceder en los supuestos de NNA que presenten un TEA, se reunirá a tales estudiantes en grupos más pequeños, separados de los cursos regulares: este contexto nos acerca a un escenario de integración.

Creemos que debe optarse por la inclusión, pues para aceptar las diferencias se debe partir por reconocer su existencia. Este diagnóstico relativo a la diferencia generalmente se logra trabajando con grupos focalizados y pequeños, labor que se puede realizar mediante la integración, con todo, es necesario obtener la incorporación progresiva de los estudiantes a la comunidad educativa, en un contexto de mayor diversidad, lo que permitiría naturalizar las diferencias y respetar las particularidades de cada alumno y/o alumna, permitiendo alcanzar el logro de los objetivos de aprendizaje por parte de todos los estudiantes. La inclusión, como opuesto de la exclusión, exige el respeto de las particularidades de cada estudiante y, al mismo tiempo, propende al logro de aprendizaje de todo el alumnado. La inclusión descansa en los principios del interés superior y de no discriminación, pues la vocación de incorporar a todos los estudiantes, tomando sus especiales condiciones en cuenta, es una manifestación de tales principios, tal como lo hemos desarrollado precedentemente.

A mayor detalle, se relaciona directamente la inclusión con el interés superior del NNA, puesto que el objetivo de este principio consiste en "garantizar el disfrute pleno y efectivo de todos los derechos reconocidos por la Convención Internacional sobre los Derechos del Niño y el desarrollo holístico del niño", así lo ha establecido el Comité de los Derechos del Niño (2013, p. 3), en su Observación General N . 14. Dicho desarrollo es físico, mental, espiritual, moral, psicológico y social, lo que se obtiene considerando todos los factores que pueden influir para que los derechos regulados por la aludida Convención, incluido el de educación, se ejerzan adecuadamente. Tengamos en vista, también, que el referido Comité, en su Observación General $N^{\circ}$. 5, ha entendido que el ejercicio efectivo del derecho a la educación solo se logra con una visión que incluya a todos y todas (COMITÉ DE LOS 
DERECHOS DEL NIÑO, 2003, p. 12). Además, se vincula la inclusión con el principio de no discriminación, porque deben existir las condiciones necesarias para que el derecho a la educación sea ejercido de manera plena por cualquier NNA, con independencia de sus diferencias y características propias. Lo anterior es un deber de los Estados Partes, según lo mandata el artículo 2 de la CDN.

En virtud de lo indicado, podemos sostener que una verdadera inclusión debe considerar una mirada de políticas públicas desde la perspectiva del interés superior del NNA y desde el principio de no discriminación. Afirmamos que la educación inclusiva, como toda educación, debe propender al desarrollo integral del estudiante y, tal como lo afirma Molina, se basa en los mismos fundamentos que inspiran a la educación regular, esto es, todos los NNA de un determinado recinto educacional deben aprender juntos, con abstracción de sus condiciones personales, sociales o culturales (MOLINA, 2015, p. 150). En esta senda, el interés superior se construye a partir de las realidades particulares y de contextos concretos relativos a cada NNA, y esas realidades necesariamente deben ser abordadas desde un enfoque de no discriminación, cumpliendo lo anterior, avanzamos en el camino que conduce a una verdadera inclusión de los NNA que tienen un TEA.

Debemos recordar que el Comité de los Derechos del Niño es consciente de la complejidad existente en la tarea de lograr una verdadera inclusión. Así, en su Observación General $\mathrm{N}^{\circ} .9$, reconoce que numerosos NNA son objeto de discriminación en el aspecto educativo, y agrega que la discriminación en este ámbito (y en sede de prestaciones de salud y de servicios sociales) repercute de manera sumamente negativa en el interés de estos, dado que conduce a negarles oportunidades de desarrollo en el futuro. Lamentablemente, Chile no escapa al panorama descrito, dado que en nuestro país se han producido casos de evidentes discriminaciones que afectan a NNA con TEA.

Con respecto a lo último, se ha expulsado a estudiantes con un TEA, particularmente vulnerables, de sus respectivos establecimientos educacionales, aunque se ha intentado disimular el verdadero motivo de dicha sanción. A mayor abundamiento, en el considerando $9^{\circ}$ de la sentencia rol $n^{\circ}$ 21.087-2020, la Corte Suprema, tribunal que conociendo la apelación de un recurso de protección rechazado por la Corte de Apelaciones de Copiapó que declaró ilegal el acto de expulsión de un niño con un TEA (el recurrido no contaba con un PIE) que cursaba la educación preescolar, indicó que:

Desprendiéndose de estas conclusiones que la decisión impugnada por esta vía es una soterrada sanción por el insuficiente desarrollo de las habilidades académicas definidas y esperadas por la recurrida respecto al educando. (CORTE SUPREMA DE CHILE, 2020)

Es muy llamativo el caso citado porque, además de lo indicado, debemos tener presente la Resolución Exenta $N^{\circ} .860$ de fecha 26 de noviembre de 2018, texto que aprueba la Circular de la Superintendencia de Educación de Chile (2018, p. 7) sobre Reglamentos Internos 
de los Establecimientos Educacionales Parvularios y que dispone en el numeral 2.8, bajo el título "Legalidad", que: "los establecimientos de Educación Parvularia no pueden contemplar sanciones a los párvulos en los Reglamentos Internos”.

En definitiva, como podemos advertir, en sede de no discriminación y de satisfacción del interés superior de NNA con TEA, aún nos queda, como país, mucho camino que recorrer. Por lo señalado, es sumamente necesario profundizar en una auténtica inclusión, puesto que, discriminaciones tan evidentes como la recientemente comentada, continúan generándose en el sistema educacional chileno.

\section{CONCLUSIONES}

En virtud del trabajo realizado, hemos podido concluir que, considerando las exigencias emanadas del principio del interés superior y las características propias del TEA, los NNA que presentan dicha condición precisan de una educación acorde a sus particulares necesidades educativas, aspecto de vital importancia para efectos de su desarrollo en condiciones de igualdad. Por lo anterior, los colegios y escuelas que reciben aportes del Estado, no pueden rechazar el ingreso de un NNA que tenga un TEA. Por el contrario, deben organizarse de tal manera que proporcionen a los NNA una educación integral focalizada en sus NEE: en dicho sentido, debe resaltarse la relevancia de los PIE. Los establecimientos educacionales que no reciban financiamiento estatal no están obligados a matricular a NNA con un TEA, la valoración de su existencia debe realizarse al momento de analizar la correspondiente admisión y no podrá con posterioridad esgrimirse el hecho de tener un estudiante con TEA, salvo casos excepcionales, como fundamento de una resolución de no renovación de matrícula.

Profundizando en la misma senda de razonamiento, los colegios y escuelas que reciban un financiamiento estatal, en conformidad a lo establecido en la Ley $\mathrm{N}^{\mathrm{o}} 20.845$ y en la Ley $\mathrm{N}^{\mathrm{o}}$. 20.422, deberán de manera necesaria realizar los ajustes pertinentes en sus planes de desarrollo de manera tal de posibilitar una adecuada educación a los estudiantes que presenten algún tipo de NEE. En consecuencia, también respecto de tales establecimientos, la mera existencia de un TEA no puede justificar con arreglo a derecho la imposición de la sanción de expulsión.

No obstante, los esfuerzos realizados para responder a las particulares necesidades educativas de los NNA con un TEA, es probable que no sean exitosos los resultados del proceso de enseñanza-aprendizaje. En dicho escenario, debemos razonar con respecto a las estrategias hasta el momento aplicadas y, eventualmente, decidir su reformulación. Así, deberá optarse preferentemente por mantener al NNA en la comunidad educativa de la que ha formado parte, y solo como ultima ratio recurrir a su derivación a un establecimiento especializado. A ello nos conduce el respeto del principio del interés superior, el que requiere evitar los cambios que puedan afectar negativamente a un NNA, cambios que, en el supuesto que estudiamos, incluso, podrían generar un agravamiento de los efectos propios del TEA. 
Súmese a lo señalado que proponemos una primacía de la "inclusión” por sobre la "integración”. En efecto, mientras que la segunda se caracteriza por un apoyo principalmente dirigido al NNA de que se trate, la primera plantea una meta más ambiciosa al reconocer la necesidad de otorgar un tratamiento más omnicomprensivo, lo que se traduce en un sistema en el que la comunidad educativa en su conjunto se organiza y adopta las medidas que resulten eficaces para acoger en su seno a los NNA que presenten una NEE. Lo último requiere un cambio de enfoque, cuestión que usualmente exige el transcurso de un período apreciable de tiempo, con todo, entendemos que la sugerida primacía es lo más conveniente para una adecuada respuesta a los requerimientos planteados por el principio del interés superior del NNA.

\section{REFERENCIAS}

ARAVENA, Paz. Apoyos emancipadores y participativos: Ruta para lograr la inclusión (educativa) de la infancia con discapacidad intelectual Fundamentos. In: ESPEJO, Nicolás; LATHROP, Fabiola (coord.) Discapacidad intelectual y Derecho. Santiago:Thomson Reuters, 2019. p. 321-349.

ARTIGAS-PALLARÈS, Josep; PAULA, Isabel. El autismo 70 años después de Leo Kanner y Hans Asperger. Revista Especializada Neuropsiquiatría, v. 115, n. 32, p. 567-587, 2011. doi: 10.4321/S021157352012000300008

ASTUDILlO, Constanza; MONDACA, Alexis. El trastorno de espectro autista. Necesidades educativas a la luz del interés superior del niño a propósito de la sentencia de la Corte de Apelaciones de Antofagasta, de 6 de diciembre de 2018. Revista de Derecho (Concepción), v. 88, n. 247, p. 257-278, 2020. doi: 10.29393/RD247-9CATE20009

BAÑA, Manoel. El rol de la familia en la calidad de vida y la autodeterminación de las personas con trastorno del espectro del autismo. Ciencia Psicológica, v. 9, n. 2, p. 323-336, 2015.

BARCIA, Rodrigo. La evolución de la custodia unilateral conforme a los principios de interés superior del niño y corresponsabilidad de los padres. Ius et Praxis, v. 2, p. 469-512, 2018.

BLANCO, Rosa. La equidad y la inclusión social: uno de los desafíos de la educación y la escuela hoy. Revista Iberoamericana sobre Calidad, Eficacia y Cambio en Educación, v. 4, n. 3, p. 1-15, 2006. 
BONILlA, María Fernanda; CHASKEL, Roberto. Trastorno del Espectro Autista. Curso Continuo de Actualización de Pediatría: Sociedad Colombiana de Pediatría, v. 15, n. 1, p. 19-29, 2016.

COMITÉ DE DERECHOS ECONÓMICOS, SOCIALES Y CULTURALES. Observación General Nº. 13 sobre el derecho a la educación (artículo 13 del Pacto). CRC/C/GC/14, 1999. Disponible en: https: / / undocs.org/es/E/C.12/1999/10. Acceso en: 16 de mayo de 2021.

COMITÉ DE LOS DERECHOS DEL NIÑO. Observación General No . 1. Párrafo 1 del artículo 29: Propósitos de la educación. CRC/C/GC/1, 2001. Disponible en: https: / undocs.org/es/CRC/GC/ $2001 / 1$. Acceso en: 16 de mayo de 2021.

COMITÉ DE LOS DERECHOS DEL NIÑO. Observación General No . 5. Medidas generales de aplicación de la Convención sobre los Derechos del Niño. CRC/GC/2003/5, 2003. Disponible en: https: / / undocs.org/es/CRC/GC/2003/5. Acceso en: 16 de mayo de 2021.

COMITÉ DE LOS DERECHOS DEL NIÑO. Observación General Nº. 9. Los derechos de los niños con discapacidad. CRC/C/GC/9, 2006. Disponible en: https://undocs.org/es/CRC/C/GC/9. Acceso en: 16 de mayo de 2021.

COMITÉ DE LOS DERECHOS DEL NIÑO. Observación General No 14 sobre el derecho del niño a que su interés superior sea una consideración primordial (artículo 3, párrafo 1). CRC/C/GC/14, 2013. Disponible en: https: / / undocs.org/es/CRC/C/GC/14. Acceso en: 16 de mayo de 2021.

CONGRESO NACIONAL DE CHILE. Decreto con Fuerza de Ley $N^{o}$ 2. Fija texto refundido, coordinado y sistematizado del Decreto con Fuerza de Ley $N^{\circ} 2$ de 1996, sobre subvención del Estado a establecimientos educacionales. Ministerio de Educación, 1998. Disponible en: http: / /bcn.cl/2k2xd. Acceso en: 16 de mayo de 2021.

CONGRESO NACIONAL DE CHILE. Ley $N^{\circ}$ 20.422. Establece normas sobre igualdad de oportunidades $e$ inclusion social de personas con discapacidad, 2010a. Disponible en: http://bcn.cl/2f7s1. Acceso en: 16 de mayo de 2021.

CONGRESO NACIONAL DE CHILE. Decreto con Fuerza de Ley $N^{o}$ 2. Fija el texto refundido, coordinado y sistematizado de la Ley $N^{\circ} 20.370$ con las normas no derogadas del Decreto con Fuerza de Ley $N^{o}$ 1, de 2005. Ministerio de Educación, 2010b. Disponible en: http://bcn.cl/2f6yy. Acceso en: 16 de mayo de 2021.

CONVENCIÓN DE LOS DERECHOS DEL NIÑO. Asamblea General de la Organización de las Naciones Unidas, 1989. Disponible en: https://www.ohchr.org/sp/professionalinterest/pages/crc. aspx. Acceso en: 26 de octubre de 2021. 
CONVENCIÓN DE LOS DERECHOS DE LAS PERSONAS CON DISCAPACIDAD. Asamblea General de la Organización de las Naciones Unidas, 2008. Disponible en: https://www.un.org/esa/ socdev/enable/documents/tccconvs.pdf. Acceso en: 26 de octubre de 2021.

CORNEJO, Juan. Nuevos excluidos en el sistema educacional chileno: problemas y desafíos. Páginas de Educación, v. 12, n. 1, p. 28-48, 2019. doi: https://doi.org/10.22235/pe.v12i1.1766

CORTE DE APELACIONES DE ANTOFAGASTA. Sentencia rol n 3149-2018. N. con Fundación Educacional Chuquicamata, 2018.

CORTE DE APELACIONES DE ARICA. Sentencia rol n 506-2019. D. con Establecimiento Educacional Miramar, 2019.

CORTE DE APELACIONES DE SAN MIGUEL. Sentencia rol nº 3936-2020. W. con Colegio Altazor de Puente Alto, 2020.

CORTE DE APELACIONES DE SANTIAGO. Sentencia rol nº 82.836-2014. E. con Escuela Especial L.A.I.J, 2015.

CORTE DE APELACIONES DE VALDIVIA. Sentencia rol nº 86-2015. E. con Instituto Alemán de Valdivia, 2015.

CORTE SUPREMA DE CHILE. Sentencia rol nº 1550-2019. N. con Fundación Educacional Chuquicamata, 2019a.

CORTE SUPREMA DE CHILE. Sentencia rol no 18.810-2019. D. con Establecimiento Educacional Miramar, 2019b.

CORTE SUPREMA DE CHILE. Sentencia rol n² 21.087-2020. X. con Scuola Italiana Giussepe Verdi, 2020 .

FLORES, Juan Carlos. Derecho a la educación: su contenido esencial en el derecho chileno. Estudios Constitucionales, v. 12, n. 2, p. 109-136, 2014. https: / /dx.doi.org/10.4067/S0718-52002014000200005.

GÓMEZ DE LA TORRE, Maricruz. El interés superior del niño. Gaceta Jurídica, v. 328, p. 23-26, 2000.

HERVÁS, Amaia; RUEDA, Isabel. Alteraciones de conducta en los trastornos del espectro autista. Revista de Neurología, v. 66, n. 1, p. 31-38, 2018. https://doi.org/10.33588/rn.66S01.2018031. 
HOLZ, Mauricio. Admisión escolar en Chile. Mecanismo de asignación de vacantes, 2019. Disponible en: https://www. camara.cl/verDoc.aspx?prmID=169430\&prmTIPO=DOCUMENTOCOMISION. Acceso en: 16 de mayo de 2021.

JUARROS, María Fernanda. ¿Educación superior como derecho o como privilegio? Las políticas de admisión a la universidad en el contexto de los países de la región. Andamios, v. 3, n. 5, p. 69-90, 2006.

LATHROP, Fabiola. Cuidado personal de los hijos. Análisis de la Ley de Matrimonio Civil y Tribunales de Familia. Santiago: Punto Lex S.A, 2005.

LEPÍN, Cristián. Reforma a las relaciones paternos-filiales: Análisis de la Ley $\mathrm{N}^{\circ}$ 20.680. Revista de Derecho. Escuela de Postgrado. Universidad de Chile, v. 3, p. 285-308, 2013.

LÓPEZ-CONTRERAS, Rony. Interés superior de los niños y niñas: definición y contenido. Revista Latinoamericana de Ciencias Sociales, Niñez y Juventud, v. 13, n. 1, p. 51-70, 2015. doi: https://doi.org/ $10.11600 / 1692715 x .1311210213$

LOVERA, Domingo. Los derechos de los niños, una orientación y un límite. $N^{o}$ 3: Igualdad y no discriminación de niños, niñas y adolescentes: necesidad de un sistema de garantías reforzadas. UNICEF, 2015. Disponible en: https:/ / www.unicef.org/chile/media/1996/file/los_derechos_de_los_ninos_una_orientacion_y_un_limite. pdf. Acceso en: 16 de mayo de 2021.

LOVERA, Domingo. Libertad de expresión e interés superior del niño, a propósito de la sentencia de la Corte de Apelaciones de Antofagasta de 6 de marzo de 2009 y Corte Suprema de 23 de abril de 2009. Revista Chilena de Derecho Privado, n. 12, p. 215-225, 2009. doi: https:/ /dx.doi.org/10.4067/S0718-80722009000100007

MARTÍNEZ, Agustín; PIQUERAS, José. Conductas repetitivas en personas con trastorno del espectro autista: relación con los antecedentes familiares y el tratamiento farmacológico. Revista Nacional e Internacional de Educación Inclusiva, v. 1, n. 10, p. 199-210, 2017.

MIRALLES, Pedro Pablo. El secuestro internacional de menores y su incidencia en España. Especial consideración del Convenio de la Haya de 1980. Madrid: Ministerio de Asuntos Sociales, 1989.

MOLINA, Yasna. Necesidades educativas especiales, elementos para una propuesta de inclusión educativa a través de la investigación acción participativa: el caso de la Escuela México. Estudios Pedagógicos, v. 41, n. especial, p. 147-167, 2015. doi: https://dx.doi.org/10.4067/S0718-07052015000300010

NÚÑEZ, Carmen Gloria et al. Una mirada desde la inclusión al Programa de Integración Escolar (PIE) en escuelas rurales chilenas: un análisis de casos. Revista Colombiana de Educación, v. 79, p. 347-368, 2020. doi: https://doi.org/10.17227/rce.num79-9725 
ORREGO, Juan Andrés. Temas de Derecho de familia. Santiago: Editorial Jurídica Metropolitana, 2007.

PEÑA, Mónica. Análisis crítico de discurso del Decreto 170 de Subvención Diferenciada para Necesidades Educativas Especiales: El diagnóstico como herramienta de gestión. Psicoperspectivas, v. 12, n. 2, p. 93-103, 2013. Disponible en: https://scielo.conicyt.cl/pdf/psicop/v12n2/art10.pdf. Acceso en: 3 de enero 2021.

RAVETLLAT, Isaac; PINOCHET, Ruperto. El interés superior del niño en el marco de la Convención Internacional sobre los Derechos del Niño y su configuración en el Derecho Civil chileno. Revista Chilena de Derecho, v. 42, n. 3, p. 903-934, 2015. doi: https: / /dx.doi.org/10.4067/S0718-34372015000300007

ROCA, Encarna. El interés del menor como factor de progreso y unificación del Derecho internacional privado. Revista Jurídica de Cataluña, v. 4, p. 915-992, 1994.

RODRÍGUEZ, María Sara. El cuidado personal de niños y adolescentes en la familia separada: criterios de resolución de conflictos de intereses entre padres e hijos en el nuevo Derecho de familia Chileno. Revista Chilena de Derecho, v. 36, n. 3, p. 545-586, 2009. doi: https://dx.doi.org/10.4067/S0718-34372009000300005

SILVEIRA, Israel. La aplicación del interés superior del niño en el ámbito educativo. Las concepciones de los profesionales de los equipos de orientación escolar. In: VIII Congreso Internacional de Investigación y Práctica Profesional en Psicología XXIII - Jornadas de Investigación. XII Encuentro de Investigadores en Psicología del MERCOSUR. Buenos Aires: Facultad de Psicología de la Universidad de Buenos Aires, 2016. Disponible en: https:// www.aacademica.org/000-044/455. Acceso en: 16 de mayo de 2021.

SUPERINTENDENCIA DE EDUCACIÓN DE CHILE. Circular que imparte instrucciones sobre Reglamentos Internos de Establecimientos de Educación Parvularia, 2018. Disponible en: https://n9.cl/n7rpu. Acceso en: 16 de mayo de 2021.

\section{Cómo CITAR ESTE ARTícuLO:}

ASTUDILLO MEZA, Constanza; MONDACA MIRANDA, Alexis. El interés superior en la trayectoria educativa de los niños, niñas y adolescentes con trastorno del espectro autista en Chile. Revista Direito GV, São Paulo, v. 18, n. 1, jan./abr. 2022, e2207. https://doi.org/ 10.1590/2317-6172202207
Constanza Astudillo Meza

Magíster en Derecho por la Universidad Católica del Norte. Profesora de Derecho Civil, Universidad Santo TOMÁS, SEDE ANTOFAGASTA. castudillomasantotomas.cl

\section{Alexis Mondaca Miranda}

Doctor en Derecho por la Pontificia Universidad Católica de Valparaíso. Profesor de Derecho Civil, Universidad de Talca.

alexis.mondacalautalca.cl 